\section{Inhibition of Respiration at Sub-optimum Substrate Concentrations}

THE following note on respiration systems arose out of some calculations made for Mr. G. D. Greville in connexion with measurements of tissue respiration at the Middlesex Hospital, London. The case considered here is that with the substrate concentration less than the optimum and with carbon monoxide present as a respiratory inhibitor. The resulting equations are different from those originally obtained by Warburg 1 , but, in correspondence, he has agreed with the present results.

We consider a homogeneous system respiring in a medium containing oxygen, carbon monoxide, and substrate.

In the absence of carbon monoxide, let $A_{s}$ be the respiration with substrate concentration $s$, less than the optimum, and $A$ the respiration with substrate concentration equal to or greater than the optimum.

In the presence of carbon monoxide, let $A_{s}^{\prime}$ and $A^{\prime}$ be the values corresponding to $A_{g}$ and $A$. We now define

so that

$$
\begin{array}{rlrl}
\varepsilon & =A_{s} / A ; & \varepsilon^{\prime}=A_{s}^{\prime} / A^{\prime} ; \\
n_{s}=A_{s}^{\prime} / A_{s} ; . & n & n=A^{\prime} / A ;
\end{array}
$$

$$
\varepsilon^{\prime} n=\varepsilon n_{\S} .
$$

Let $\sigma_{\mathrm{O}_{2}}, \sigma_{\mathrm{co}}$, and $\sigma_{g}$ be those fractions of the catalyst which are combined with oxygen, carbon monoxide, and substrate respectively. When oxygen only is present, the respiration depends directly on $\sigma_{s}$, provided that the oxygen partial pressure is not less than a certain minimum value, which is always exceeded in practice, that is,

$$
\sigma_{s}=\varepsilon \text {. }
$$

When oxygen and carbon monoxide are present, residual respiration/inhibited respiration equals

$$
n_{s} /\left(1-n_{s}\right)=\sigma_{\mathrm{O}_{2}}^{\delta} / \sigma_{\mathrm{CO}}^{8},
$$

where $\sigma_{\mathrm{O}_{2}}^{8}$ is that fraction of the whole catalyst which is in combination with oxygen and substrate simultaneously, and similarly for $\sigma_{\mathrm{CO}}^{8}$. If the sum of the oxygen and carbon monoxide partial pressures is not less than a certain minimum value, then $\sigma_{\mathrm{O}_{2}}+\sigma_{\mathrm{CO}}=1$, and this is the case in practice. We assume that the probability of finding an oxygen and a substrate molecule simultaneously in combination with a given catalyst molecule is equal to the product of the separate probabilities of finding oxygen or substrate molecules in combination with that same catalyst molecule, and similarly for carbon monoxide. That is, we assume that the distributions of both oxygen and carbon monoxide over the catalyst are independent of the distribution of substrate. Then

and similarly

$$
\sigma_{0_{2}}^{s}=\varepsilon \sigma_{O_{2}},
$$

$\sigma_{\mathrm{CO}}^{8}=\varepsilon \sigma_{\mathrm{cO}}$.

Therefore, from (3),

$$
n_{8} /\left(1-n_{s}\right)=\sigma_{0_{3}} / \sigma_{\mathrm{co}} .
$$

Now, in the equilibrium state, rate of combination of oxygen with enzyme equals rate of dissociation of oxygen-enzyme complex plus rate of removal of oxygen by chemical reaction, that is,

$$
\lambda_{1} p_{O_{2}}\left(1-\sigma_{O_{2}}-\sigma_{\mathrm{CO}}\right)=\lambda_{2} \sigma_{O_{2}}+\lambda_{3} \sigma \sigma_{O_{2}} ;
$$

and similarly for carbon monoxide,

$$
\mu_{1} p_{\mathrm{CO}}\left(1-\sigma_{\mathrm{O}_{2}}-\sigma_{\mathrm{CO}}\right)=\mu_{2} \sigma_{\mathrm{CO}},
$$

where $p_{\mathrm{O}_{2}}$ and $p_{\mathrm{CO}}$ are the pressures of oxygen and carbon monoxide in the solution, respectively, and $\lambda_{1}, \lambda_{2}, \lambda_{3}, \mu_{1}, \mu_{2}$ are constants.

Now, from equations (2), (4), (5), and (6), we have

$$
\frac{n_{8}}{1-n_{s}}=\frac{\sigma_{\mathrm{O}_{2}}}{\sigma_{\mathrm{CO}}}=\frac{p_{\mathrm{O}_{3}}}{p_{\mathrm{CO}}} \cdot \frac{a}{b+\varepsilon},
$$

where $a=\lambda_{1} \mu_{2} / \mu_{1} \lambda_{3}$ and $b=\lambda_{2} / \lambda_{3}$.

Also, from (1), we have

$$
\frac{n_{s}}{1-n_{s}}=\frac{\varepsilon^{\prime} n}{\varepsilon-\varepsilon^{\prime} n} \text {. }
$$

It is evident from (7) that when the substrate concentration is diminished so that the system becomes unsaturated with respect to substrate, the respiration is less markedly depressed by carbon monoxide only when $\varepsilon$ is not negligible in comparison with $b$. That is, when the rate of removal of oxygen by chemical reaction cannot be neglected in comparison with the rate of dissociation of oxygenenzyme complex, or, in terms of the constants of the system, $\varepsilon \lambda_{3}$ is comparable in value with $\lambda_{2}$.

Publication of this result has been delayed many months because it was thought that an opportunity for verification might occur. As this has not been possible, the result is put forward in the hope that other workers in this field might be able to test it.

$$
\text { W. F. Floyd. }
$$

Department of Physiology,

Middlesex Hospital Medical School, London, W.1.

March 8.

${ }^{1}$ Biochem. Z., 189, 354 (1927).

\section{Photodynamic Hæmolysis by Carcinogenic Hydrocarbons}

IN recent publications, Mottram and Doniach ${ }^{1,2,3,4}$ reported that many carcinogenic hydrocarbons have a photodynamic effect upon Paramecia and upon the skin of mice. These hydrocarbons under the same experimental conditions did not cause photodynamic hæmolysis of erythrocytes.

This report deals with experiments made in order to find out whether by using a longer time of exposure, and a different technique of preparing the colloidal solutions of the three carcinogenic hydrocarbons, 3:4 benzpyrene, 1:2:5:6 dibenzanthracene, and methylcholanthrene, photodynamic hæmolysis could be obtained. (The carcinogens used were from the Union Chimique Belge 'Meurice'.)

The colloidal solutions of the hydrocarbons were prepared in the following way : 20 c.c. of isotonic saline solution were poured into a large Petri dish of approximately $30 \mathrm{~cm}$. diameter. To this were added 10 c.c. of an acetone $1: 10,000$ hydrocarbon solution; the whole was left at room temperature, until all the acetone evaporated. The Petri dish was slightly shaken at the beginning in order to spread the solution all over the bottom. When the acetone had evaporated, the solution was poured into a test tube, care being taken to include all traces of hydrocarbon deposited on the wall of the Petri dish in the solution. This solution, 